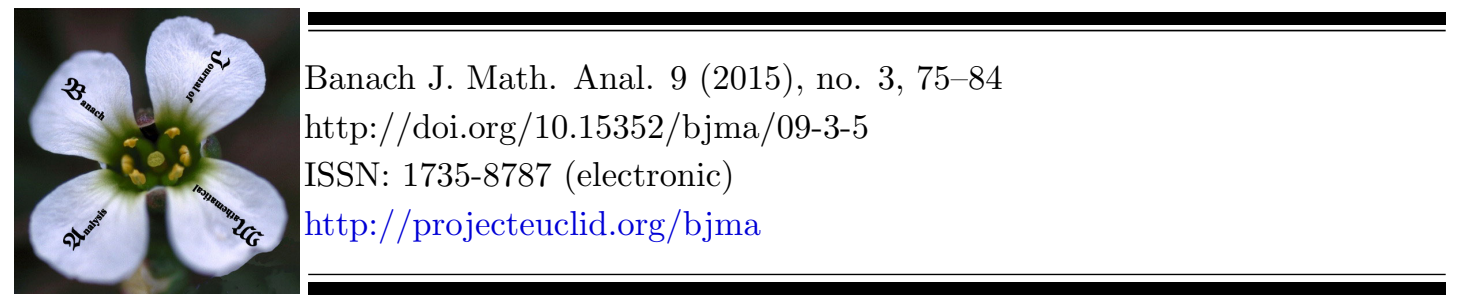

\title{
PERIPHERALLY MULTIPLICATIVE OPERATORS ON UNITAL COMMUTATIVE BANACH ALGEBRAS
}

\author{
M. NAJAFI TAVANI
}

Communicated by K. Jarosz

\begin{abstract}
Let $T: A \longrightarrow B$ be a surjective operator between two unital semisimple commutative Banach algebras $A$ and $B$ with $T 1=1$. We show that if $T$ satisfies the peripheral multiplicativity condition $\sigma_{\pi}(T f . T g)=\sigma_{\pi}(f . g)$ for all $f$ and $g$ in $A$, where $\sigma_{\pi}(f)$ shows the peripheral spectrum of $f$, then $T$ is a composition operator in modulus on the Silov boundary of $A$ in the sense that $|f(x)|=|T f(\tau(x))|$, for each $f \in A$ and $x \in \partial(A)$ where $\tau: \partial(A) \longrightarrow \partial(B)$ is a homeomorphism between Šilov boundaries of $A$ and $B$.
\end{abstract}

\section{INTRODUCTION}

Assume that $A$ and $B$ are algebras of continuous functions on Hausdorff spaces $X$ and $Y$ respectively. A map $T: A \longrightarrow B$ is called multiplicatively spectrumpreserving if $s p_{B}(T(f) \cdot T(g))=s p_{A}(f . g)$, for all $f$ and $g$ in $A$, where $s p_{A}(f)$ shows the spectrum of an element $f$ in $A$. If the spectrums of the elements of the algebras $A$ and $B$ are bounded subsets of the plane then $T: A \longrightarrow B$ is called peripherally multiplicative if $\sigma_{\pi}(T(f) \cdot T(g))=\sigma_{\pi}(f . g)$, for all $f$ and $g$ in $A$, where $\sigma_{\pi}(f)$, the peripheral spectrum of $f$, is defined by $\sigma_{\pi}(f)=\left\{\lambda \in s p_{A}(f):|\lambda|=\right.$ $\left.\max _{z \in s p_{A}(f)}|z|\right\}$.

In 2002 Molnar [14] proved the following: If $X$ is a first-countable compact Hausdorff space, $A=C(X)$, the space of all continuous functions on $X$ and $T: A \longrightarrow A$ a multiplicatively spectrum-preserving surjection, then $T(1)$ is a signum function, i.e., $(T(1))^{2}=1$, and there exists a homeomorphism $\tau$ of $X$ onto

Date: Received: Apr. 9, 2014; Accepted: Sep. 30, 2014.

2010 Mathematics Subject Classification. Primary 46J10; Secondary 46J20, 47B48.

Key words and phrases. Banach function algebra, peaking function, Shilov boundary, peripheral spectrum, peripherally multiplicative operator. 
itself such that $T(f)(x)=T(1)(x) f(\tau(x))$ for all $x \in X$ and $f \in C(X)$. Molnar's Theorem motivated new researches about operators between algebras of continuous functions which preserves multiplicatively the norm, range or spectrum of algebra elements. For example in [5] authors extended Molnar's Theorem to unital commutative semisimple Banach algebras. Some other extensions of Molnar's Theorem to diverse classes of Banach algebras can be found in $[4,8,15,16]$.

In 2006 Luttman and Tonev showed that it is not necessary that the entire spectrum be preserved in order to completely determine the structures of function algebras:

Theorem 1.1. [13] Let $A$ and $B$ be uniform algebras on compact Hausdorff spaces $X$ and $Y$ respectively. If $T: A \longrightarrow B$ is a surjective operator such that $\sigma_{\pi}(T(f) . T(g))=\sigma_{\pi}(f . g)$, for all $f$ and $g$ in $A$, then $T(1)$ is a signum function and there exists a homeomorphism $\tau: c(B) \longrightarrow c(A)$ such that $T(f)(x)=$ $T(1)(x) f(\tau(x))$ for all $x \in c(B)$ and $f \in A$, where $c(A)$ denotes the Choquet boundary of $A$

The proof of the above Theorem depends basically on the fact that the Choquet boundary $c(A)$ of a uniform algebra $A$ consists exactly of strong boundary points of $A$. So extensions of the Theorem to unital commutative semisimple Banach algebras are not always possible. On the other hand, if every point in the maximal ideal space is a strong boundary point, then positive results exists. For instance, in [10] it is proven that any unital peripherally multiplicative surjection between Lipschitz algebras is an algebra isomorphism. Also there is a similar result for additive peripherally multiplicative surjections between dense subsets of uniform algebras [18].

In 2007 Lambert, Lutman and Tonev showed that Theorem 1.1 is valid under a weaker condition $\sigma_{\pi}(T(f) . T(g)) \cap \sigma_{\pi}(f . g) \neq \emptyset$ if, in addition, $T$ preserves the peaking functions [11]. There are another extensions of Theorem 1.1 which can be found in $[9,10,18]$.

In [6] authors raised a question " Is any unital peripherally multiplicative surjection between unital semisimple commutative Banach algebras an isomorphism?". In this paper by generalizing some of the results in [13] to Banach function algebras and using the upper semicontinuity of the peripheral spectrum function, we show that a peripherally multiplicative operator $T: A \longrightarrow B$ between two unital commutative semisimple Banach algebras $A$ and $B$ is injective. Also we show that if $T$ is, in addition, unital and surjective then it is a composition operator in modulus on the Šilov boundary of $A$ and induces a homeomorphism between Šilov boundaries of $A$ and $B$.

\section{PRELIMINARIES}

Given a compact Hausdorff space $X$, a function algebra $A$ on $X$ is a subalgebra of $C(X)$ which contains the constants and separates the points of $X$ in the sense that for each $x, y \in X$ with $x \neq y$ there exists a function $f \in A$ such that $f(x) \neq f(y)$. A Banach function algebra $A$ on $X$ is a function algebra on $X$ which is a Banach algebra with respect to some norm. If the norm of a Banach function algebra $A$ on a compact Hausdorff space $X$ is the sup-norm, i. e., if 
$\|f\|_{X}=\sup _{x \in X}|f(x)|$ then $A$ is called a uniform algebra on $X$. If $A$ is a function algebra on $X$, then $\bar{A}$, the uniform closure of $A$, is a uniform algebra on $X$.

For a Banach function algebra $A$ on a compact Hausdorff space $X$ there exists a natural embedding of $X$ into $\mathrm{M}_{A}$, the maximal ideal space of $A$, through the map $J: x \longmapsto \varphi_{x}$, where $\varphi_{x}$ is the evaluation homomorphism at $x \in X$. We say that $A$ is a natural Banach function algebra on $X$ if the map $J$ is also surjective.

If $A$ is a unital commutative semisimple Banach algebra then the Gelfand transform $\Gamma: A \longrightarrow \hat{A} \subseteq C\left(\mathrm{M}_{A}\right) ; f \longmapsto \hat{f}$ is an algebra isomorphism. So identifying $A$ with $\hat{A}$ each unital commutative semisimple Banach algebra $A$ can be considered as a natural Banach function algebra on its maximal ideal space.

Let $A$ be a function algebra on a compact Hausdorff space $X$. A function $f$ in $A$ is a peaking function of $A$ if $\|f\|_{X}=1$ and $|f(x)|<1$ for each $x \in X$ with $f(x) \neq 1$. For a peaking function $f \in A$, the set $P(f)=\{x \in X: f(x)=1\}$ is called the peaking set (or peak set) of $f$. We denote the set of all peaking functions of $A$ by $\mathcal{F}(A)$. Also for a fixed point $x \in X$ the set of all peaking functions $f \in A$ with $x \in P(f)$ is denoted by $\mathcal{F}_{x}(A)$. For an element $f \in A$, the maximal set $M_{f}$ of $f$ is defined by $M_{f}=\left\{x \in X:|f(x)|=\|f\|_{X}\right\}$. It is easy to see that for each $f \in A$, if $|f(x)|=\|f\|_{X}$ for some $x \in X$ then the function $g=\frac{1}{2}\left(1+\frac{f}{f(x)}\right)$ is a peaking function with $P(g) \subseteq M_{f}$. A point $x \in X$ is a strong boundary point for $A$ if for every neighborhood $V$ of $x$ there exists an $f \in A$ such that $\|f\|_{X}=f(x)=1$ and $|f(y)|<1$ if $y \in X \backslash V$. If we consider $\frac{1}{2}(1+f)$ instead of $f$, we can assume that the function $f$ in the later definition is a peaking function with $x \in P(f) \subseteq V$. A subset $E$ of $X$ is called a boundary for $A$ if every $f \in A$ assumes its maximum modulus at some point of $E$. The unique minimal closed boundary for $A$, which exists by [17, Theorem 7.4] is called the Silov boundary for $A$ and is denoted by $\partial(A)$. It is clear that $x \in X$ is in $\partial(A)$ if and only if for each neighborhood $V$ of $x$ there exists $f \in A$ such that $M_{f} \subset V$, or, in other words, there exists a peaking function $f$ in $A$ with $P(f) \subset V$.

If $A$ is a commutative Banach algebra then $\hat{A}$ is a function algebra on $\mathrm{M}_{A}$ and we shall refer to the Šilov boundary for $\hat{A}$ as the Šilov boundary for $A$.

The Choquet boundary $c(A)$ of a function algebra $A$ on a compact Hausdorff space $X$ is the set of all $x \in X$ for which $\varphi_{x}$ is an extreme point of the unit ball of the dual space of $\left(A,\|\cdot\|_{X}\right)$, so clearly $c(A)=c(\bar{A})$. Also it is known that for a function algebra $A, \partial A$ is the closure of $c(A)$ [1]. If $A$ is a uniform algebra on a compact Hausdorff space $X$ then it is well known that $c(A)$ consists of all strong boundary points for $A$ [17, Theorem 7.30].

In the following we state Bishop's Lemma for function algebras. A similar result is proven in [19]

Lemma 2.1. Let $A$ be a function algebra on a compact Hausdorff space $X$ and $E \subseteq X$ be a peak set for $A$. For each $f \in A$ with $\left.f\right|_{E} \neq 0$ there exists a peaking function $h \in \mathcal{F}(\bar{A})$ such that $P(h)=E$ and

i) $|f(z) h(z)|<\max _{x \in E}|f(x)|$ for each $z \in X \backslash E$,

ii) $h$ is a uniform limit of a sequence $\left\{h_{n}\right\}$ in $\mathcal{F}(A)$ with $P\left(h_{n}\right)=E$. 
Proof. Without loss of generality we may assume that $\|f\|_{E}=\max _{x \in E}|f(x)|=1$. As in [12, pag 161], Consider a peaking function $g \in A$ with $P(g)=E$ and open sets $U_{n}=\left\{x \in X:|f(x)|<1+\frac{1}{2^{n}}\right\}$, each of them containing $E$. So for each $n \in \mathbb{N}$ there exists $p_{n} \in \mathbb{N}$ such that $\max _{X \backslash U_{n}}\left|g^{p_{n}}\right|<\frac{1}{2^{n}\|f\|_{X}}$. Let $g_{n}=g^{p_{n}}$ and set $h=\sum_{k=1}^{\infty} \frac{g_{k}}{2^{k}}$. Since $\left\|g_{n}\right\|_{X}=1$, the series converges uniformly and hence $h \in \bar{A}$. An argument similar to [12, page 161] shows that $h$ is a peaking function in $\bar{A}$ with $P(h)=E$ and, moreover, $f h$ attains its maximum modulus exclusively within $E$.

To prove (ii) note that each $g_{n}$ is a peaking function in $A$ and $P\left(g_{n}\right)=E$. Set $s_{n}=\sum_{k=1}^{n} \frac{g_{k}}{2^{k}}$ and $\alpha_{n}=\sum_{k=1}^{n} \frac{1}{2^{k}}$. It is easy to see that $h_{n}=\frac{1}{\alpha_{n}} s_{n}$ is a sequence in $\mathcal{F}(A)$ converging uniformly to $h$ and for each $n \in \mathbb{N}, P\left(h_{n}\right)=E$.

\section{PERIPHERAL SPECTRUM OF FUNCTION ALGEBRA'S ELEMENTS}

In this Section by generalizing some of the results in [13] and using the upper semicontinuity of the peripheral spectrum function, we show that any peripherally multiplicative operator between two unital commutative semisimple Banach algebras is injective.

The following Lemma follows from [19, Lemma 2.2] but we state it for the sake of completness.

Lemma 3.1. Let $A$ be a function algebra on a compact Hausdorff space $X$ and $f, g \in A$. If $\|f h\|_{X} \leq\|g h\|_{X}$ for all peaking functions $h \in A$, then $|f(x)| \leq|g(x)|$ on $\partial(A)$.

Proof. For the proof we modify the proof of [13, Lemma 2]. Suppose on the contrary that there exists $x_{0} \in \partial(A)$ such that $\left|g\left(x_{0}\right)\right|<\left|f\left(x_{0}\right)\right|$. Choose $\delta>0$ such that $\left|g\left(x_{0}\right)\right|<\delta<\left|f\left(x_{0}\right)\right|$, and open neighborhoods $V_{1}$ and $V_{2}$ of $x_{0}$ in $X$ such that $|g(x)|<\delta$ on $V_{1}$ and $|f(x)|>\delta$ on $V_{2}$. Set $V=V_{1} \cap V_{2}$. Since $x_{0} \in \partial(A)$, there exists $h \in \mathcal{F}(A)$ such that $P(h) \subseteq V$. By choosing a sufficiently high power of $h$ we may assume that $\|g h\|_{X}<\delta$. Obviously for each $z \in P(h)$ we have

$$
\|g h\|_{X}<\delta<|f(z) h(z)| \leq\|f h\|_{X}
$$

which contradicts the hypothesis.

Corollary 3.2. Let $A$ be a function algebra on a compact Hausdorff space $X$ and $f, g \in A$. If $\|f h\|_{X}=\|g h\|_{X}$ for all peaking functions $h \in A$, then $|f(x)|=|g(x)|$ on $\partial(A)$.

In the next Lemma, following the same method as in [2, Theorem 3.4.2] we show that the peripheral spectrum function $f \mapsto \sigma_{\pi}(f)$ is upper semicontinuous on a unital commutative Banach algebra.

Lemma 3.3. Let $A$ be a unital commutative Banach algebra with unit 1. Then the peripheral spectrum function $f \mapsto \sigma_{\pi}(f)$ is upper semicontinuous on $A$, that is for every open set $U$ containing $\sigma_{\pi}(f)$ there exists $\delta>0$ such that $\|f-g\|<\delta$ implies that $\sigma_{\pi}(g) \subseteq U$. 
Proof. We first note that $\sigma_{\pi}(f)=\left\{\lambda \in s p_{\mathrm{A}}(f):|\lambda|=\max _{z \in s p_{\mathrm{A}}(f)}|z|=\|\hat{f}\|_{M_{\mathrm{A}}}\right\}$, for each $f \in A$. Now suppose on the contrary that there exist sequences $\left\{g_{n}\right\}$ in $\mathrm{A}$ and $\left\{\alpha_{n}\right\}$ in $\mathbb{C}$ such that $\lim _{n \rightarrow \infty} g_{n}=f$ and $\alpha_{n} \in \sigma_{\pi}\left(g_{n}\right) \cap(\mathbb{C} \backslash U)$. Since $\left|\alpha_{n}\right|=\left\|\hat{g}_{n}\right\|_{M_{\mathrm{A}}}$ and $\lim _{n \rightarrow \infty}\left\|\hat{g}_{n}\right\|_{M_{\mathrm{A}}}=\|\hat{f}\|_{M_{\mathrm{A}}}$, the sequence $\left\{\alpha_{n}\right\}$ is bounded and consequently it has a limit point $\alpha$. Without loss of generality we may assume that $\left\{\alpha_{n}\right\}$ converges to $\alpha$. Clearly $\alpha \notin U$. Now if $\alpha \notin s p_{\mathrm{A}}(f)$ then $\alpha \mathbf{1}-f$ is invertible and so $\alpha_{n} \mathbf{1}-g_{n}$ will be invertible for $n$ large enough, which is a contradiction. Therefore $\alpha \in s p_{\mathrm{A}}(f)$. On the other hand the equation $|\alpha|=\|\hat{f}\|_{M_{\mathrm{A}}}$ implies that $\alpha \in \sigma_{\pi}(f) \subseteq U$ which is again a contradiction.

Remark 3.4. i) Let $A$ be a Banach function algebra on a compact Hausdorff space $X$. In [7] it is shown that if $A$ is natural then $M(A)=M(\bar{A})$. This clearly implies that $s p_{A}(f)=s p_{\bar{A}}(f)$, for each $f \in A$. Consequently, for a natural Banach function algebra $A$ on a compact Hausdorff space $X$, the peripheral spectrums of an element $f \in A$ with respect to both algebras $A$ and $\bar{A}$ are the same. Note that for each $f \in \bar{A}, \sigma_{\pi}(f)=\operatorname{Ran}_{\pi}(f)$, where $\operatorname{Ran}_{\pi}(f)$ is the peripheral range of $f$, that is, the set $\operatorname{Ran}_{\pi}(f)=f(X) \cap\left\{z \in \mathbb{C}:|z|=\|f\|_{X}\right\}$ [13, Lemma 1];

ii) If $A$ and $B$ are natural Banach function algebras on compact Hausdorff spaces $X$ and $Y$, respectively, and $T: A \longrightarrow B$ is a peripherally multiplicative operator then $\|T(f) T(g)\|_{Y}=\|f g\|_{X}$ for each $f, g \in A$, since $\sigma_{\pi}(f)=\operatorname{Ran}_{\pi}(f)$ and $T$ is peripherally multiplicative. Also it is easy to see that if $T$ is unital and surjective then $T(\mathcal{F}(A))=\mathcal{F}(B)$.

Lemma 3.5. Let $A$ be a natural Banach function algebra on $X$. Let $f, g \in A$ and $\left\{h_{n}\right\}$ be a sequence in $A$ which converges to an element $h$ in $\bar{A}$. If $\sigma_{\pi}\left(f h_{n}\right) \cap$ $\sigma_{\pi}\left(g h_{n}\right) \neq \emptyset$, for each $n \in \mathbb{N}$, then $\sigma_{\pi}(f h) \cap \sigma_{\pi}(g h) \neq \emptyset$

Proof. Suppose on the contrary that $\sigma_{\pi}(f h) \cap \sigma_{\pi}(g h)=\emptyset$. So there exist disjoint open sets $U$ and $V$ containing $\sigma_{\pi}(f h)$ and $\sigma_{\pi}(g h)$, respectively. Upper semicontinuity of the peripheral spectrum function shows that there exists $N \in \mathbb{N}$ such that $\sigma_{\pi}\left(f h_{n}\right) \subseteq U$ and $\sigma_{\pi}\left(g h_{n}\right) \subseteq V$, for each $n \geqslant N$. But this contradicts to the fact that $\sigma_{\pi}\left(f h_{n}\right) \cap \sigma_{\pi}\left(g h_{n}\right) \neq \emptyset$.

Using the above Lemma we can modify the proof of [13, Lemma 3] and get the following result:

Lemma 3.6. Let $A$ be a natural Banach function algebra on a compact Hausdorff space $X$ and $f, g \in A$. If $\sigma_{\pi}(f h)=\sigma_{\pi}(g h)$ for all peaking functions $h \in A$, then $f=g$ on $X$.

Proof. Obviously $\|f h\|_{X}=\|g h\|_{X}$ for all peaking functions $h \in A$. So by corollary 3.2 we have $|f(x)|=|g(x)|$, for each $x \in \partial(A)$. Let $x_{0} \in \partial(A)$. If $f\left(x_{0}\right)=0$ then clearly $f\left(x_{0}\right)=g\left(x_{0}\right)$. Otherwise choose a neighborhood $V_{1}$ of $x_{0}$ such that $f \neq 0$ on $V_{1}$. For an arbitrary neighborhood $V$ of $x_{0}$ there exists a function $k \in \mathcal{F}(A)$ such that $P(k) \subseteq V \cap V_{1}$. By Lemma 2.1 we can find a function $h \in \mathcal{F}(\bar{A})$ with the same peaking set as $k$, so that $f h$ attains its maximum modulus exclusively within $P(h)$ and $h$ is a uniform limit of a sequence $\left\{h_{n}\right\}$ of peaking functions in $A$ with $P\left(h_{n}\right)=P(k)$, for each $n \in \mathbb{N}$. In fact we can choose $h \in \mathcal{F}(\bar{A})$ such that both functions $f h$ and $g h$ attain their maximum modulus exclusively within $P(h)$. By 
hypothesis $\sigma_{\pi}\left(f h_{n}\right)=\sigma_{\pi}\left(g h_{n}\right)$ for each $n \in \mathbb{N}$. This implies, by Lemma 3.5, that $\sigma_{\pi}(f h) \cap \sigma_{\pi}(g h) \neq \emptyset$. Let $z \in \sigma_{\pi}(f h) \cap \sigma_{\pi}(g h)$. Then $|z|=\|f h\|_{X}=\|g h\|_{X}$ and there exists $x_{1}, x_{2} \in X$ such that $z=f h\left(x_{1}\right)=g h\left(x_{2}\right)$. Obviously $x_{1}, x_{2} \in P(h)$, by the choice of $h$, and therefore $f\left(x_{1}\right)=g\left(x_{2}\right)$. Since each neighborhood of $x_{0}$ contains points $x_{1}$ and $x_{2}$ such that $f\left(x_{1}\right)=g\left(x_{2}\right)$, we have $f\left(x_{0}\right)=g\left(x_{0}\right)$ by the continuity of $f$ and $g$. So $f=g$ on $\partial(A)$ and Consequently $f=g$ on $X$.

Using Lemma 3.6 and an argument similar to [13, Proposition 1], we get the following corollary:

Corollary 3.7. Let $A$ and $B$ be natural Banach function algebras on compact Hausdorff spaces $X$ and $Y$, respectively. Then every peripherally multiplicative operator $T: A \longrightarrow B$ is injective.

\section{MAin RESUlts}

Let $A$ be a Banach function algebra on a compact Hausdorff space $X$. Consider the collection $\Omega$ of subsets of $X$ which are nonempty intersection of peak sets for $A$ and order $\Omega$ by inclusion.

Definition 4.1. [3, Definition 4.1] A member of $\Omega$ which is minimal with respect to the ordering is called a minimal set for $A$ on $X$. The collection of all minimal sets on $X$ is denoted by $\Sigma_{X}$.

Obviously $\Sigma_{X}$ is a collection of pairwise disjoint closed subsets of $X$. If $Q \in \Sigma_{X}$ then every peak set either contains $Q$ or is disjoint from it. So clearly for each

$x \in Q$ we have $Q=\bigcap_{f \in \mathcal{F}_{x}(A)} P(f)$. Since $X$ is compact and every member of $\Sigma_{X}$ is closed, it follows from finite intersection property and Zorn's Lemma that every peak set contains a minimal set and consequently every maximal set contains a minimal set. A trivial minimal set is a minimal set which contains only one point. A point $x \in X$ is a strong boundary point for $A$ if and only if $\{x\}$ is a trivial minimal set. Each minimal set in a uniform algebra is trivial but [3, example 4.3] shows that non-trivial minimal sets exist.

Definition 4.2. Let $\left\{Q_{\nu}\right\}_{\nu \in I}$ be a net in $\Sigma_{X}$. we say:

i) $x \in X$ is a limit point of $\left\{Q_{\nu}\right\}_{\nu \in I}$ or $\left\{Q_{\nu}\right\}_{\nu \in I}$ converges to $x$ if for each neighborhood $U$ of $x$ there exists $\nu \in I$ such that $Q_{\omega} \subseteq U$ whenever $\omega>v$.

ii) $x \in X$ is an accumulation point of $\left\{Q_{\nu}\right\}_{\nu \in I}$ or $\left\{Q_{\nu}\right\}_{\nu \in I}$ accumulates at $x$ if there exists a subnet $\left\{Q_{\alpha}\right\}_{\alpha \in J}$ of $\left\{Q_{\nu}\right\}_{\nu \in I}$ and a net $\left\{x_{\alpha}\right\}_{\alpha \in J}$ such that for each $\alpha \in J, x_{\alpha} \in Q_{\alpha}$ and $\left\{x_{\alpha}\right\}_{\alpha \in J}$ converges to $x$.

We denote the set of all accumulation points of a net $\left\{Q_{\nu}\right\}_{\nu \in I}$ in $\Sigma_{X}$ by $E_{\left\{Q_{\nu}\right\}_{\nu \in I}}$. It is easy to see that a net $\left\{Q_{\nu}\right\}_{\nu \in I}$ converges to an element $x \in X$ if and only if $E_{\left\{Q_{\nu}\right\}_{\nu \in I}}=\{x\}$

Lemma 4.3. For each $x \in \partial(A)$ there exists a net in $\Sigma_{X}$ converging to $x$. Also if $x$ is not a strong boundary point for $A$ then we can choose the mentioned net such that it consists of infinitely many elements of $\Sigma_{X}$. 
Proof. If $x$ is a strong boundary point then the singleton $\{x\}$ itself is a minimal set. Otherwise there exists an open neighborhood $U$ of $x$ such that for each $f \in \mathcal{F}(A)$ if $P(f) \subseteq U$ then $x \notin P(f)$. Consider the set

$$
I=\{\nu: \nu \text { is an open neighbourhood of } x \text { contained in } U\} .
$$

Obviously $I$ is a directed set with respect to the inverse inclusion. Since $x \in \partial(A)$, each neighborhood of $x$ contains a peak set and consequently contains a minimal set. So for each $\nu \in I$ there exists a $Q_{\nu} \in \Sigma_{X}$ with $Q_{\nu} \subset \nu$. Clearly $\left\{Q_{\nu}\right\}_{\nu \in I}$ converges to $x$. Let $\Lambda=\left\{Q_{\nu}: \nu \in I\right\}$. If $\Lambda$ is a finite set then $\nu^{\prime}=U \backslash \bigcup_{\nu \in I} Q_{\nu}$ belongs to $I$ and $Q_{\nu^{\prime}} \notin \Lambda$, which is a contradiction.

Remark 4.4. (i) Let $x \in \partial(A)$ and $\left\{Q_{\nu}\right\}_{\nu \in I}$ be a net converging to $x$ which consists of finitely many elements in $\Sigma_{X}$. By Definition 4.2 one can see that there exists $\nu \in I$ such that $Q_{\omega}=Q_{\nu}$ whenever $\omega>\nu$ and $Q_{\nu}=\{x\}$. So we conclude that $x \in \partial(A)$ is strong boundary point for $A$ if and only if there exists a net converging to $x$ which consists of finitely many elements in $\Sigma_{X}$.

(ii) Let $\left\{Q_{\nu}\right\}_{\nu \in I}$ be an arbitrary net in $\Sigma_{X}$. Since for each $\nu \in I$ the minimal set $Q_{\nu}$ intersects $c(A)=c(\bar{A})$ and $\partial(A)$ is a compact subset of $X$ containing $c(A)$, so $E_{\left\{Q_{\nu}\right\}_{\nu \in I}} \cap \partial(A)$ is a nonempty subset of $X$. Note that if $\left\{Q_{\nu}\right\}_{\nu \in I}$ consists of finitely many elements in $\Sigma_{X}$ then

$E_{\left\{Q_{\nu}\right\}_{\nu \in I}}=\cup\left\{Q_{\nu}\right.$ : there exists a subnet $\left\{Q_{\omega}\right\}_{\omega \in J}$ of $\left\{Q_{\nu}\right\}_{\nu \in I}$ such that $Q_{\omega}=$ $Q_{\nu}$ for each $\left.\omega \in J\right\}$.

Our main result is the following:

Theorem 4.5. Let $A$ and $B$ be natural Banach function algebras on compact Hausdorff spaces $X$ and $Y$ respectively, and $T: A \longrightarrow B$ be a unital peripherally multiplicative surjection. Then there exists a homeomorphism $\tau: \partial(A) \longrightarrow \partial(B)$ such that $|f(x)|=|T f(\tau(x))|$ for each $f \in A$ and $x \in \partial(A)$.

Proof. The proof of the Theorem is divided into the following steps. The proofs of step1 and step2 is omitted since they are similar to the proofs of [8, Lemma 3.7], [8, Lemma 3.10] and [8, Lemma 3.9].

step1. If $x$ is an element of a minimal set for $A$ on $X$ then $\bigcap_{f \in \mathcal{F}_{x}(A)} P(T f)$ is a minimal set for $B$ on $Y$.

step2. Let $\psi: \Sigma_{X} \longrightarrow \Sigma_{Y}$ be the function defined by $\psi(Q)=\bigcap_{f \in \mathcal{F}_{x}(A)} P(T f)$ where $x$ is an arbitrary element of $Q$. Then $\psi$ is a bijection and $\|f\|_{Q}=\|T f\|_{\psi(Q)}$ holds for all $Q \in \Sigma_{X}$ and $f \in A$, where $\|f\|_{Q}$ is the sup-norm of $f$ on $Q$ as a compact subset of $X$.

step3. Let $x_{0} \in \partial(A)$ and $\left\{Q_{\nu}\right\}_{\nu \in I}$ be a net in $\Sigma_{X}$ converging to $x_{0}$. Suppose $y_{0} \in E_{\left\{\psi\left(Q_{\nu}\right)\right\}_{\nu \in I}}$ and $k \in B$. Then for each $r>0,\left|k\left(y_{0}\right)\right|>r$ implies that $\left|T^{-1}(k)\left(x_{0}\right)\right|>r$.

Choose $r^{\prime}>0$ such that $\left|k\left(y_{0}\right)\right|>r^{\prime}>r$ and a neighborhood $V$ of $y_{0}$ in $Y$ such that $|k(y)|>r^{\prime}$ on $V$. By Definition 4.2 there exists a subnet $\left\{\psi\left(Q_{\beta}\right)\right\}_{\beta \in J}$ of $\left\{\psi\left(Q_{\nu}\right)\right\}_{\nu \in I}$ and a net $\left\{y_{\beta}\right\}_{\beta \in J}$ such that for each $\beta \in J, y_{\beta} \in \psi\left(Q_{\beta}\right)$, and $\left\{y_{\beta}\right\}_{\beta \in J}$ converges to $y_{0}$. So we can find $\alpha \in J$ such that $y_{\beta} \in V$ whenever $\beta>\alpha$. By step2 for each $\beta>\alpha$ we have $\left\|T^{-1}(k)\right\|_{Q_{\beta}}=\|k\|_{\psi\left(Q_{\beta}\right)} \geq\left|k\left(y_{\beta}\right)\right|>r^{\prime}$. Since the net $\left\{Q_{\beta}\right\}_{\beta \in I}$ converges to $x_{0}$ we have $\left|T^{-1}(k)\left(x_{0}\right)\right| \geq r^{\prime}>r$ 
step4. Let $x_{0} \in \partial(A)$ and $\left\{Q_{\nu}\right\}_{\nu \in I}$ be a net in $\Sigma_{X}$ converging to $x_{0}$. Suppose $y_{0} \in E_{\left\{\psi\left(Q_{\nu}\right)\right\}_{\nu \in I}} \cap \partial B$. If $\left\{P_{\alpha}\right\}_{\alpha \in \Gamma}$ is a net in $\Sigma_{Y}$ converging to $y_{0}$ then $E_{\left\{\psi^{-1}\left(P_{\alpha}\right)\right\}_{\alpha \in \Gamma}}=\left\{x_{0}\right\}$.

By Remark 4.4, $E_{\left\{\psi^{-1}\left(P_{\alpha}\right)\right\}_{\alpha \in \Gamma}} \cap \partial(A)$ is a nonempty subset of $X$. Now suppose that there exists $x^{\prime} \in E_{\left\{\psi^{-1}\left(P_{\alpha}\right)\right\}_{\alpha \in \Gamma}}$ such that $x^{\prime} \neq x_{0}$. since $A$ is a function algebra on $X$, there exists an $f \in A$ such that $f\left(x^{\prime}\right)=1$ and $f\left(x_{0}\right)=0$. Fix two positive real numbers $r$ and $r^{\prime}$ such that $0<r<r^{\prime}<1$. There exists an open neighborhood $V$ of $x^{\prime}$ such that $|f(x)|>r^{\prime}>r$ on $V$ and $x_{0} \in X \backslash V$. Since $x^{\prime} \in E_{\left\{\psi^{-1}\left(P_{\alpha}\right)\right\}_{\alpha \in \Gamma}}$, there exists a subnet $\left\{\psi^{-1}\left(P_{\beta}\right)\right\}_{\beta \in J}$ of $\left\{\psi^{-1}\left(P_{\alpha}\right)\right\}_{\alpha \in \Gamma}$ and a net $\left\{x_{\beta}^{\prime}\right\}_{\beta \in J}$ in $X$ such that $x_{\beta}^{\prime} \in \psi^{-1}\left(P_{\beta}\right)$ for each $\beta \in J$ and $\left\{x_{\beta}^{\prime}\right\}_{\beta \in J}$ converges to $x^{\prime}$. So we can find $\gamma \in J$ such that $x_{\beta}^{\prime} \in V$ whenever $\beta \in J$ and $\beta>\gamma$. Therefore for each $\beta \in J$ with $\beta>\gamma$ we have $\|T f\|_{P_{\beta}}=\|f\|_{\psi^{-1}\left(P_{\beta}\right)} \geq\left|f\left(x_{\beta}^{\prime}\right)\right|>r^{\prime}>r$. Note that $\left\{P_{\beta}\right\}_{\beta \in J}$ is a subnet of $\left\{P_{\alpha}\right\}_{\alpha \in \Gamma}$ and so converges to $y_{0}$. Therefore $\left|T f\left(y_{0}\right)\right| \geq r^{\prime}>r$. On the other hand $y_{0} \in E_{\left\{\psi\left(Q_{\nu}\right)\right\}_{\nu \in I}}$ so by previous step $\left|T^{-1}(T f)\left(x_{0}\right)\right|=\left|f\left(x_{0}\right)\right|>r$ which is a contradiction since $f\left(x_{0}\right)=0$. Hence $x^{\prime}=x_{0}$.

Note that if $y_{0}$ is a strong boundary point for $B$ then we can choose $\left\{P_{\alpha}\right\}_{\alpha \in \Gamma}$ such that $P_{\alpha}=\left\{y_{0}\right\}$ for each $\alpha \in \Gamma$ so by Remark 4.4 we have $E_{\left\{\psi^{-1}\left(P_{\alpha}\right)\right\}_{\alpha \in \Gamma}}=$ $\psi^{-1}\left(\left\{y_{0}\right\}\right)=\left\{x_{0}\right\}$. This shows that, in this case, $x_{0}$ is in fact a strong boundary point for $A$.

step5. Let $x_{0} \in \partial(A)$ and $\left\{Q_{\nu}\right\}_{\nu \in I}$ be a net in $\Sigma_{X}$ converging to $x_{0}$. Then the set $E_{\left\{\psi\left(Q_{\nu}\right)\right\}_{\nu \in I}}$ is a singleton that belongs to $\partial(B)$. Furtheremore if $x_{0}$ is a strong boundary point for $A$ then $\psi\left(\left\{x_{0}\right\}\right)$ is a singletone which consists of a strong boundary point for $B$.

By Remark 4.4 $E_{\left\{\psi\left(Q_{\nu}\right)\right\}_{\nu \in I}} \cap \partial B \neq \emptyset$. Let $y_{0} \in E_{\left\{\psi\left(Q_{\nu}\right)\right\}_{\nu \in I}} \cap \partial B$. Suppose on the contrary that there exists $y_{1} \in E_{\left\{\psi\left(Q_{\nu}\right)\right\}_{\nu \in I}}$ such that $y_{1} \neq y_{0}$. Since $B$ is a function algebra, we can find $k \in B$ such that $k\left(y_{0}\right)=0$ and $k\left(y_{1}\right)=1$. For each $\varepsilon>0$ consider a neighbourhood $V$ of $y_{0}$ such that $|k(y)|<\varepsilon$ on $V$. Now by Lemma 4.3 there exists a net $\left\{P_{\alpha}\right\}_{\alpha \in J}$ in $\Sigma_{Y}$ converging to $y_{0}$. So we can find $\alpha \in J$ such that for each $\beta \in J$ if $\beta>\alpha$ then $\left\|T^{-1}(k)\right\|_{\psi^{-1}\left(P_{\beta}\right)}=\|k\|_{P_{\beta}}<\varepsilon$. By the previous step $E_{\left\{\psi^{-1}\left(P_{\alpha}\right)\right\}_{\alpha \in J}}=\left\{x_{0}\right\}$ so $\left|T^{-1}(k)\left(x_{0}\right)\right| \leq \varepsilon$. On the othere hand $1=\left|k\left(y_{1}\right)\right|>r$, for some $1>r>\varepsilon$. So by step3, $\left|T^{-1}(k)\left(x_{0}\right)\right|>r>\varepsilon$ which is a contradiction.

Now suppose that $x_{0}$ is a strong boundary point for $A$. We can choose $\left\{Q_{\nu}\right\}_{\nu \in I}$ such that $Q_{\nu}=\left\{x_{0}\right\}$ for each $\nu \in I$. So by Remark $4.4 E_{\left\{\psi\left(Q_{\nu}\right)\right\}_{\nu \in I}}=\psi\left(\left\{x_{0}\right\}\right)$ and since the minimal set $\psi\left(\left\{x_{0}\right\}\right)$ is a singleton, by the first part of the proof, so it consists of a strong boundary point for $B$.

step6. Let $x \in \partial A$. If $\left\{Q_{\nu}\right\}_{\nu \in I}$ and $\left\{Q_{\nu}^{\prime}\right\}_{\nu \in J}$ are two different nets converging to $x$ then $E_{\left\{\psi\left(Q_{\nu}\right)\right\}_{\nu \in I}}=E_{\left\{\psi\left(Q_{\nu}^{\prime}\right)\right\}_{\nu \in J}}$

Let $E_{\left\{\psi\left(Q_{\nu}\right)\right\}_{\nu \in I}}=\left\{y_{0}\right\}$ and $E_{\left\{\psi\left(Q_{\nu}^{\prime}\right)\right\}_{\nu \in J}}=\left\{y_{1}\right\}$. Suppose on the contrary that $y_{0} \neq y_{1}$. Consider the function $k \in B$ such that $k\left(y_{0}\right)=0$ and $k\left(y_{1}\right)=1$. Fix $\varepsilon>0$. There exists $\alpha \in I$ such that $\left\|T^{-1}(k)\right\|_{Q_{\beta}}=\|k\|_{\psi\left(Q_{\beta}\right)}<\varepsilon$ whenever $\beta \in I$ and $\beta>\alpha$. Therefore $\left|T^{-1}(k)(x)\right|<\varepsilon$, since $\left\{Q_{\nu}\right\}_{\nu \in I}$ converges to $x$. On the other hand step3 shows that $\left|T^{-1}(k)(x)\right|>\varepsilon$, since $1=\left|k\left(y_{1}\right)\right|>\varepsilon$, which is a contradiction. 
step7. Let $\tau: \partial(A) \longrightarrow \partial(B)$ be the function defined by $\{\tau(x)\}=E_{\left\{\psi\left(Q_{\nu}\right)\right\}_{\nu \in I}}$ where $\left\{Q_{\nu}\right\}_{\nu \in I}$ is an arbitrary net in $\Sigma_{X}$ converging to $x$. Then $\tau$ is a homeomorphism and for each $f \in A$ and $x \in \partial(A)$

$$
|f(x)|=|T f(\tau(x))| .
$$

It is easy to see that equation (4.1) is valid for each $f \in A$ and $x \in \partial(A)$, by step 2 and definition of the function $\tau$. Now let $x_{1}, x_{2} \in \partial(A)$ and $\tau\left(x_{1}\right)=$ $\tau\left(x_{2}\right)$. Clearly $T f\left(\tau\left(x_{1}\right)\right)=T f\left(\tau\left(x_{2}\right)\right)$ for each $f \in A$. So by equation (4.1), $\left|f\left(x_{1}\right)\right|=\left|f\left(x_{2}\right)\right|$ and consequently $x_{1}=x_{2}$ since $A$ is a function algebra. So $\tau$ is injective. To prove the surjectivity of $\tau$ we first note that $T$ is bijective and since the peripheral multiplicativity property is symmetric with respect to $f$ and $T(f)$, it holds also for the inverse operator $T^{-1}$. On the other hand step2 shows that $\psi$ is a bijection. Let $y \in \partial(B)$ and $\left\{P_{\nu}\right\}_{\nu \in I}$ be a net in $\Sigma_{Y}$ converging to $y$. Considering the peripheral multiplicative operator $T^{-1}: B \longrightarrow A$ and the bijection $\psi^{-1}: \Sigma_{Y} \longrightarrow \Sigma_{X}$, according to step5, $E_{\left\{\psi^{-1}\left(P_{\nu}\right)\right\}_{\nu \in I}}$ consists of just one element, say $x$, in $\partial(A)$. Obviously $\left\{\psi^{-1}\left(P_{\nu}\right)\right\}_{\nu \in I}$ converges to $x$ and so $\{\tau(x)\}=E_{\left\{\psi\left(\psi^{-1}\left(P_{\nu}\right)\right)\right\}_{\nu \in I}}=E_{\left\{P_{\nu}\right\}_{\nu \in I}}=\{y\}$, that is $\tau$ is surjective.

Now let $x \in \partial(A)$ and $\left\{x_{\alpha}\right\}_{\alpha \in I}$ be a net in $\partial(A)$ converging to $x$. Since $\partial(B)$ is compact, we can assume that $\left\{\tau\left(x_{\alpha}\right)\right\}_{\alpha \in I}$ converges to an element $y$ in $\partial(B)$. If $\tau(x) \neq y$ then we can consider a function $f \in A$ such that $T f(\tau(x))=1$ and $T f(y)=0$. According to equation (4.1), $|f(x)|=1$. Let $r>0$. Since $\left\{x_{\alpha}\right\}_{\alpha \in I}$ converges to $x$, there exists $\alpha \in I$ such that $\left|f\left(x_{\beta}\right)\right|>r$ whenever $\beta>\alpha$. Using equation (4.1) once more we conclude that $\left|T f\left(\tau\left(x_{\beta}\right)\right)\right|>r$ for each $\beta>\alpha$. On the other hand $\left\{\tau\left(x_{\alpha}\right)\right\}_{\alpha \in I}$ converges to $y$ and $T f$ is continuous, so $|T f(y)| \geq r$ which is a contradiction. Consequently $\tau$ is continuous. If we consider $T^{-1}: B \longrightarrow A$ and the corresponding mapping $\tau^{-1}: \partial(B) \longrightarrow \partial(A)$, the same argument shows that $\tau^{-1}$ is also continuous, that is, $\tau$ is a homeomorphism.

Remark 4.6. (i) Note that if $x \in X$ is a strong boundary point for $A$ then $\tau(x) \in Y$ is a strong boundary point for $B$. Consequently if $f \in \mathcal{F}_{x}(A)$ then $T f \in \mathcal{F}_{\tau(x)}(B)$ since $\{\tau(x)\}=\psi(\{x\})=\bigcap_{f \in \mathcal{F}_{x}(A)} P(T f)$.

(ii) Suppose that $f \in A$ is invertible. By equation (4.1) we have

$$
\mid T\left(f^{-1}\right)\left(\tau(x)|=| f^{-1}(x)|=1 /| f(x)|=1 /| T f(\tau(x) \mid .\right.
$$

Consequently $\left|T\left(f^{-1}\right)(y) \cdot T f(y)\right|=1$ on $\tau(\partial(A))=\partial(B)$. On the other hand $\sigma_{\pi}\left(T\left(f^{-1}\right) \cdot T f\right)=\sigma_{\pi}\left(f^{-1} \cdot f\right)=\{1\}$. So $T\left(f^{-1}\right)(y) \cdot T f(y)=1$ on $\partial(B)$ which clearly implies that $T f$ is invertible and $T\left(f^{-1}\right)=(T f)^{-1}$.

\section{REFERENCES}

1. J. Araujo and J.J. Font, On Silove boundaries for subspaces of continuous functions, Topology Appl. 77 (1997), 79-85.

2. B. Aupetit, A primer on spectral theory, Springer-Verlag New York, Inc, 1991.

3. H.G. Dales, Boundaries and peak points for Banach function algebras, Proc. London Math. Soc. 22 (1971), 121-136.

4. O. Hatori, T. Miura and H. Takagi, Characterization of the isometric isomorphisms between uniform algebrasvia non-linear range preserving properties, Proc. Amer. Math. Soc. 134 (2006), 2923-2930. 
5. O. Hatori, T. Miura and H. Takagi, Unital and multiplicatively spectrum-preserving surjections between semi-simple commutative Banach algebras are linear and multiplivative, J. Math. Anal. Appl. 326 (2007), 281-296.

6. O. Hatori, S. Lambert, A. Luttman, T. Miura, T. Tonev and R. Yates, Spectral preservers in commutative Banach algebras, Contemp. Math. 547 (2011), 103-124.

7. T.G. Honary, Relations between Banach function algebras and their uniform closures, Proc. Amer. Math. Soc. 109 (1990), 337-342.

8. M. Hosseini and F. Sady, Multiplicatively range-preserving maps between Banach function algebras, J. Math. Anal. Appl. 357 (2009), 314-322.

9. A. Jimenez-Vargas, A. Luttman and M. Villegas-Vallecillos, Weakly peripherally multiplicative surjections of pointed Lipschitz algebras, Rocky Mountain J. Math. 40(2010), 19031922 .

10. A. Jimenez-Vargas and M. Villegas-Vallecillos, Lipschitz algebras and peripherallymultiplicative maps, Acta Math. Sinica 24 (2008), no. 8, 1233-1242.

11. S. Lambert, A. Luttman and T. Tonev, Weakly peripherally-multiplicative operators between uniform algebras, Contemp. Math. 435 (2007), 265-281.

12. G.M. Leibowitz, Lectures on complex function algebras, Scott, Foresman and company, 1970.

13. A. Luttman and T. Tonev, Uniform algebra isomorphisms and peripheral multiplicativity, Proc. Amer. Math. Soc. 135 (2007), 3589-3598.

14. L. Molnar, Some characterizations of the automorphisms of $B(H)$ and $C(X)$, Proc. Amer. Math. Soc. 130 (2002), 111-120.

15. N.V. Rao and A.K. Roy, Multiplicatively spectrum-preserving maps of function algebras, Proc. Amer. Math. Soc. 133 (2005), 1135-1142.

16. N.V. Rao and A.K. Roy, Multiplicatively spectrum-preserving maps of function algebras II, Proc. Edinb. Math. Soc. 48 (2005), 219-229.

17. E.L. Stout, The teory of uniform algebras, Bogden and Quigley, Inc. Publishers, 1971.

18. T. Tonev and E. Toneva, Composition operators between subset of function algebras, Contemp. Math. 547 (2011), 227-237.

19. T. Tonev, Weak multiplicative operators of function algebras without unit, Banach Center Publ. 91, 2010, 411-421.

Department of Mathematics, Islamshahr Branch, Islamic Azad University, IsLAMSHAHR, IRAN.

E-mail address: najafi@iiau.ac.ir 